EPJ Web of Conferences 67, 02033 (2014)

DOI: $10.1051 /$ epjconf / 20146702033

(C) Owned by the authors, published by EDP Sciences, 2014

\title{
Contact-free measurement of the flow field of a liquid metal inside a closed container
}

Christiane Heinicke, ${ }^{\text {a }}$

Department of Mechanical Engineering, Ilmenau University of Technology, D-98684 Ilmenau, Germany

\begin{abstract}
The measurement of flow velocities inside metal melts is particularly challenging. Due to the high temperatures of the melts it is impossible to employ measurement techniques that require either mechanical contact with the melt or are only adaptable to translucent fluids. In the past years a number of electromagnetic techniques have been developed that allows a contact-free measurement of volume flows. One of these techniques is the so-called Lorentz Force Velocimetry (LFV) in which the metal flow is exposed to an external, permanent magnetic field. The interaction between the metal and the magnet not only leads to a force on the fluid, but also on the magnet. The force can be measured and is proportional to the velocity of the melt. Moreover, by using a small permanent magnet it is possible to resolve spatial structures inside the flow. We will demonstrate this using a model experiment that has been investigated with different reference techniques previously. The experimental setup is a cylindrical vessel filled with a eutectic alloy which is liquid at room temperature. The liquid metal can be set into motion by means of a propeller at the top of the liquid. Depending on the direction of rotation of the propeller, the flow inside the vessel takes on different states. Beside the vessel, we place a Lorentz Force Flowmeter (LFF) equipped with a small permanent magnet. By measuring the force on the magnet at different positions and different rotation speeds, we demonstrate that we can qualitatively and quantitatively reconstruct the flow field inside the vessel.
\end{abstract}

\section{Introduction}

Industrial flows like metal melts or glass melts are typically chemically aggressive and very hot. The measurement of flow velocities inside these fluids has been a challenge until recently, before several flow meters have been developed that use electromagnetic techniques to determine the volume flux without or only indirect contact to the fluid [1-6].

One of these techniques is Lorentz Force Velocimetry (LFV, [4]), a non-contact electromagnetic method that exposes the flow to a magnetic field and uses the resulting force on the magnet to determine the velocity of the flow. LFV has been applied successfully to industrial type flows and to electrolytes in the laboratory [7-9]. All of these measurements have been volume flux measurement. Recently, it has been shown that by using a small permanent magnet LFV may provide some spatial information about the flow field. The sensor that utilizes the small magnet is called Local Lorentz Force Flowmeter, in short L2F2.

So far, the L2F2 has been used for duct flow experiments only [10]. In this paper, we will present the application of the L2F2 to a liquid metal flow in a closed cylindrical container at room temperature. The flow is driven by a propeller at the top; depending on the direction of rotation there are different states of the flow inside the cylinder. We will demonstrate how the L2F2 can be applied to this setup and how information on the velocity field inside the vessel can be inferred from measurements of the force on the magnet outside the vessel.

\footnotetext{
a e-mail: christiane.heinicke@tu-ilmenau.de
}

\section{Experimental Setup}

\subsection{The container}

We use a cylindrical container made of plexiglass with an inner diameter of $9 \mathrm{~cm}$, inner height of $16 \mathrm{~cm}$, and wall thickness of $4 \mathrm{~mm}$. The setup is shown in figure 1 . The top of the container is formed by a lid to which 8 vertical guiding blades are attached (figure 1a). A propeller below these blades drives the flow, the rotation speed is set to a maximum of $\pm 1700 \mathrm{rpm}$.

The container is filled with the eutectic alloy GaInSn which is liquid at room temperature.

\subsection{Flow inside the container}

The propeller can rotate in both directions. In the first direction, the metal is pumped downward in the middle, and in the second, the metal is pumped upward. The following flow characteristics are described in more detail in ref. [1].

In the downward pumping case the liquid is moving upwards close to the container walls, forming a toroid. Additionally, the metal is moved sideways, such that the fluid particles are moving in a spiralling manner around a toroid. At the bottom corner of the container is a smaller, counterrotating toroid.

In the case of upward pumping, the liquid is moving downwards at the container walls, forming a poloidal motion opposite to the one in the downward pumping case. However, because the metal is first being directed towards the guiding blades, its toroidal, sideways motion is mostly suppressed. In effect, both flow regimes differ by the direction of their poloidal motion and the strength of the sideways motion. 


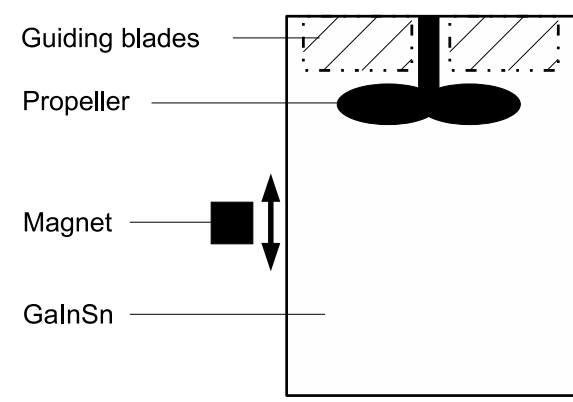

(a)

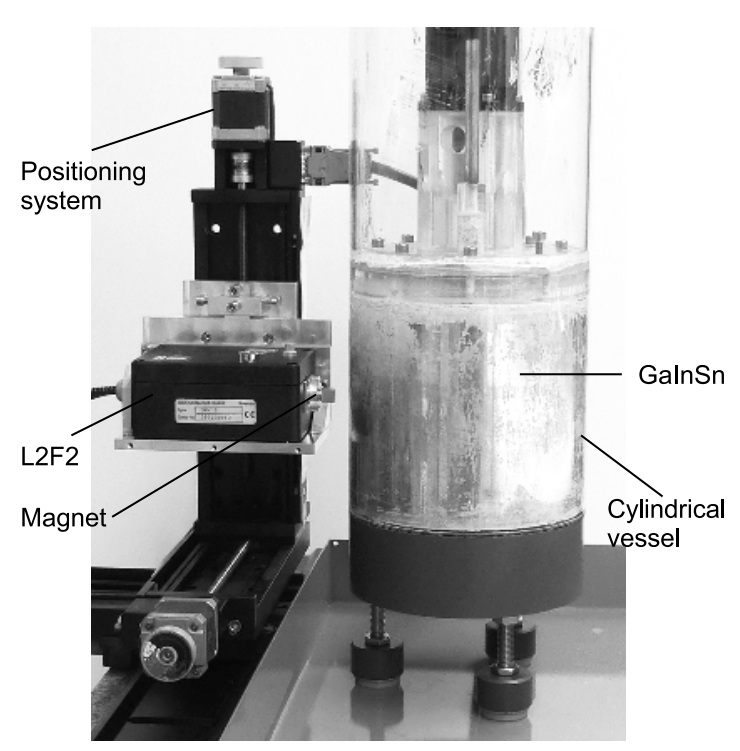

(b)

Fig. 1. Experimental setup. A plexiglass cylinder is filled with the eutectic alloy GaInSn. Below the top are vertical guiding blades; a propeller drives the flow. The magnet system is positioned outside the cylinder, almost touching the wall during measurements, and recording profiles of the horizontal and vertical force components along the side wall. (a) Sketch. (b) Photograph (the distance between magnet and cylinder is increased for better visibility).

\subsection{Force measurement system}

Our measurement system is placed outside the cylinder. It comprises a force measurement system carrying a small magnet cube of $1 \mathrm{~cm}$ edge length. On the surface, the magnet has a flux density of $475 \mathrm{mT}$.

The force measurement system (L2F2) itself is a quartz glass parallel spring whose deformation is a measure for the applied force. Despite the dead weight of the magnet of $8 \mathrm{~g}$, the resolution of the sensor is $0.3 \mu \mathrm{N}$.

At each new parameter setting, we measure the force signal for (i) the fluid at rest and (ii) the fluid moving, each for about a minute and with sufficient time gaps to ensure that the measurement is in the steady state regime.

From previous flow experiments using a square duct with GaInSn it is known that the force $F$ measured with the L2F2 depends on the mean velocity $\bar{v}$ as [11]:

$$
F=0.187 \frac{\mathrm{mN} \mathrm{s}}{\mathrm{cm}} \bar{v}-0.082 \mathrm{mN}
$$

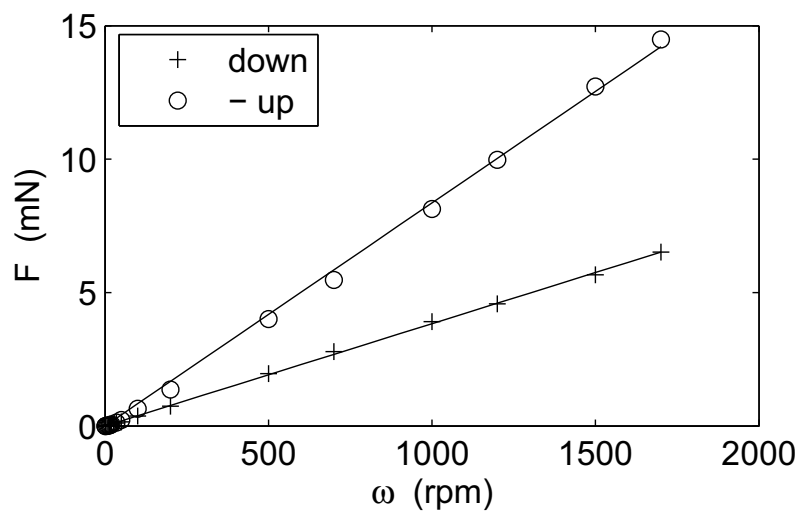

Fig. 2. Dependence of the magnitude of the vertical force component on the direction and speed of rotation of the propeller. In the downward pumping case $(+)$, the force is weaker and pointed upward. In the upward pumping case (o), the magnitude of the force is higher, but the force is directed downward ("- up").

Despite the different geometry of the flow containers, we will use equation 1 for estimating the velocities in the experiment at hand, because of the lack of calibration data for GaInSn in a cylindrical configuration.

\section{Results and Discussion}

We are interested in the dependence of the measured force on two input parameters: the rotation speed of the propeller including its direction and the position of the magnet along the cylinder. For both parameters we measured the vertical and the azimuthal force component.

\subsection{Influence of the rotation speed}

It is expected that the force on the magnet should increase with increasing rotation speed of the propeller, i.e. with an increased flow velocity inside the vessel [4].

The magnitude of the forces for the different rotation speeds can be seen in figure 2. Measurements have been taken with the magnet at the vertical location of the force maximum (also see figure 3 ). In the downward pumping case $(+)$ in figure 2 the force is positive, and only about half as strong in magnitude as the negative force in the upward pumping case (o). For both rotation directions the force on the magnet increases approximately linearly with the number of rotations per minute.

Accordingly, the horizontal force component is weak, when the vertical force component is strong (upward pumping case) and strong, when the vertical component is weak (downward pumping case), respectively. This is not shown here as a dependence on the rotation speed for the sake of compactness; however, it may be inferred from figure 4 in section 3.2.

Moreover, it is known from previous experiments that the magnitude of the flow velocity components significantly depends on the rotation direction.

In the downward pumping case, the metal flows downward unhindered in the center of the cylinder and upward at the wall, leading to an upward (positive) force on the 


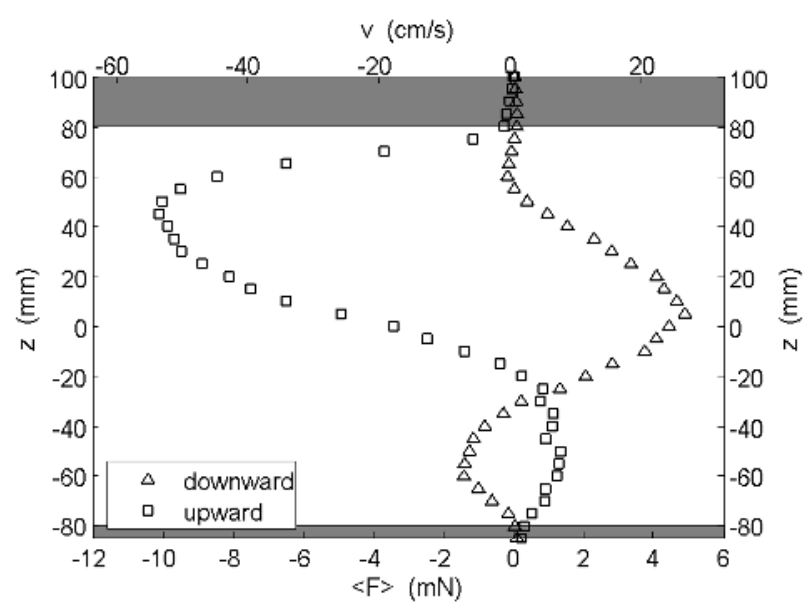

Fig. 3. L2F2 measurements for the vertical velocity component, and for both pumping directions. During downward pumping, the vertical velocity component points upward at the wall (in the positive direction). During upward pumping the vertical velocity component points downward (negative direction) and is considerably stronger than in the downward pumping case.

magnet. In addition to this poloidal movement there is a toroidal movement towards the left, seen from the magnet. Momentum from the propeller is split between both velocity components. When the propeller is turning in the opposite direction, the flow is directed upward in the middle of the cylinder. On the way towards the wall it passes the guiding blades that suppress the additional rotational movement. Therefore, in the upward pumping case the poloidal velocity component is much weaker than in the downward pumping case, and the momentum of the propeller is concentrated on the toroidal velocity component. Therefore, the vertical velocity is higher for the upward pumping case, resulting in a higher force.

\subsection{Vertical line profile}

We will now come to the dependence of the force on the magnet on the second parameter, the vertical position of the magnet. We will scan the flow inside the cylinder with the L2F2 along a vertical line outside the cylinder in steps of $5 \mathrm{~mm}$.

It has been shown before [12] that it is possible to obtain a space-dependent signal from the flow with the help of a small permanent magnet. Compared to the experimental setup in ref. [12], the dimensions of the vessel are several times larger, and thus it can be expected that the magnet cube is responding to an even smaller fraction of the total cylinder volume at each measurement position.

In figure 3 we show the profiles for the vertical force component for both the downward and upward pumping case. Note that figure 3 has two $\mathrm{x}$-axes; the upper $\mathrm{x}$-axis has been determined according to equation 1 . It can be seen that, in accordance with figure 2 , the vertical force maximum is roughly twice as large for the upward pumping case as for the downward pumping case. More importantly, beside the main maximum at or above the mid-height of the cylinder, there is a small peak of opposite sign in the bottom part of the cylinder for both pumping directions.
This implies a counter-rotating toroid, which is in perfect agreement with the flow structure found in ref. [1].

Figure 4 shows the profiles for the two force components, both for the downward pumping case (figure 4a) and for the upward pumping case (figure 4c). Graphs of the resultant velocity vectors directly at the cylinder wall are shown in figures $4 \mathrm{~b}$ and $\mathrm{d}$. One can see that the horizontal velocity component is significantly weaker than the vertical component for both cases. The direction of the horizontal (toroidal) movement is to the left, independent of the direction of rotation of the propeller. However, the magnitude of the toroidal movement is almost twice as strong for the downward pumping case as for the upward pumping case. The much weaker horizontal movement is due to the breaking effect of the guiding blades, and has also been found in [1].

\section{Conclusion and Outlook}

We have performed contact-free measurements of the liquid metal flow inside a confined container. The flow structure inside the container has been known from previous experiments. Using a Local Lorentz Force Flowmeter (L2F2) we have measured the induced force on a small permanent magnet which depends on the fluid velocity in the vicinity of the magnet. Our varied input parameters are the flow velocity and the direction of pumping, which changes the structure of the flow, and the vertical position of the magnet.

The signal of the L2F2 not only depends on the rotation speed of the propeller but also on the position of the magnet. It was therefore possible to scan the container and determine distinct flow structures. The qualitative shape of the velocity profiles we determined with our L2F2 matches the outcome of the previous experiments using other measurement techniques.

Next immediate steps include a quantitative comparison with other measurement techniques, and the extension of the force sensor to a sensor that allows the simultaneous measurement of all three force components. We also aim at improving the repeatability of our measurements and the calibration in order to apply the L2F2 as a sole velocity sensor to different experimental setups.

The experiments have been performed in close cooperation with Thomas Wondrak. The author gratefully acknowledges the funding within the framework of the Helmholtz-Alliance "Limtech".

\section{References}

1. F. Stefani, T. Gundrum, and G. Gerbeth, Phys. Rev. E 70, (2004) 056306

2. J. Priede, D. Buchenau, and G. Gerbeth, Magnetohydrodynamics 45, (2009) 451

3. J. Priede, D. Buchenau, and G. Gerbeth, Meas. Sci. Technol. 22, (2011) 055402

4. A. Thess, E. Votyakov, and Y. Kolesnikov, Phys. Rev. Lett. 96, (2006) 164501

5. S. Miralles, G. Verhille, N. Plihon, and J.-F. Pinton, Rev. Sci. Instrum. 82, (2011) 095112

6. S. Eckert, G. Gerbeth, and V.I. Melnikov, Exp. Fluids 35, (2003) 381 


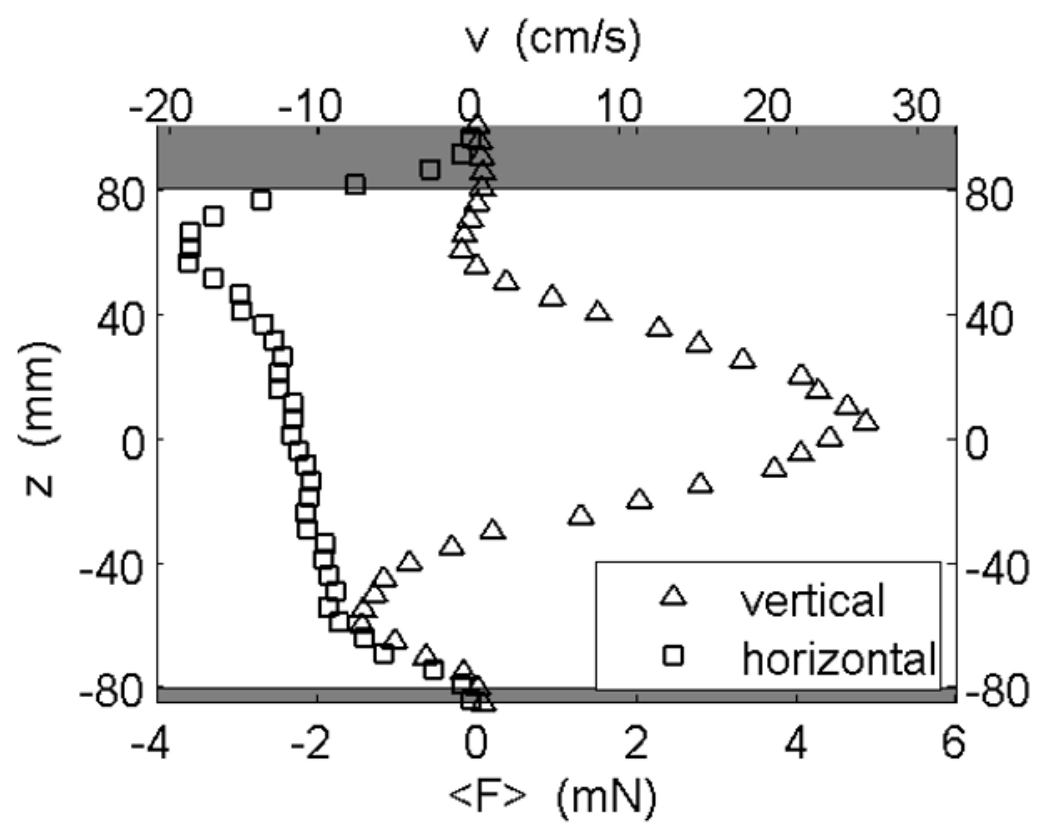

(a)

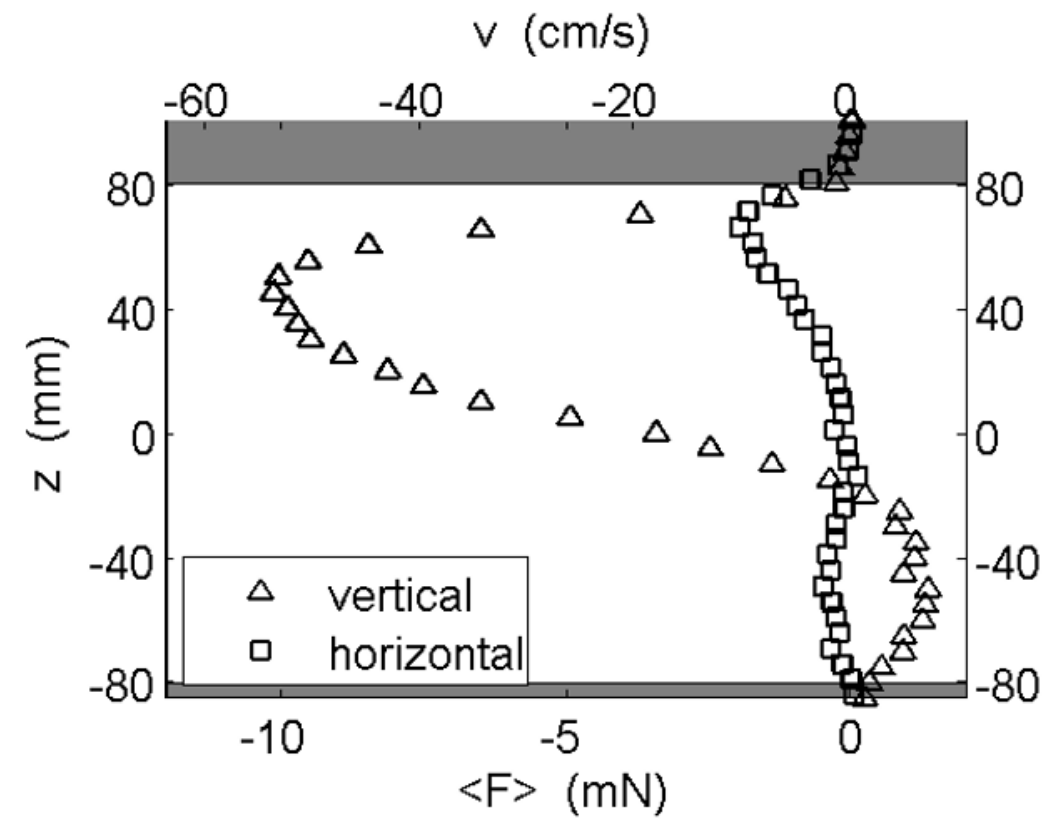

(c)

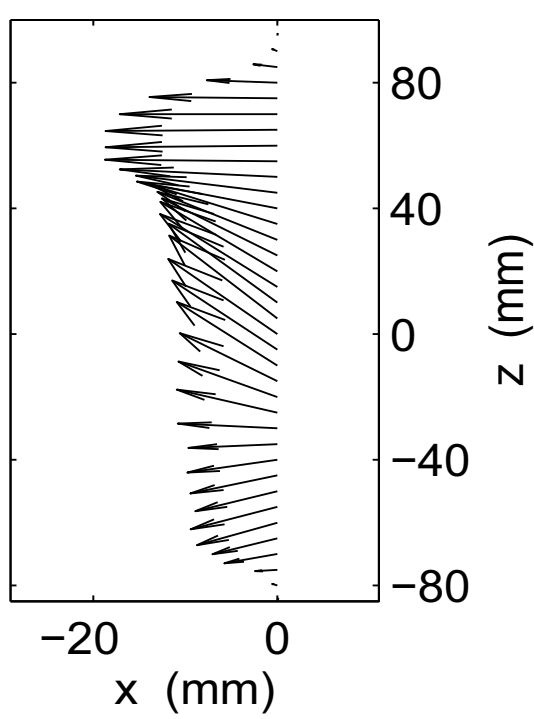

(b)

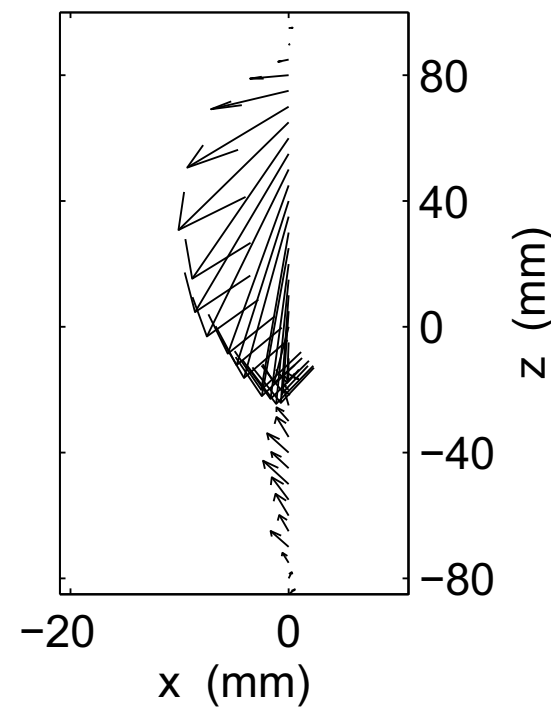

(d)

Fig. 4. Comparison of vertical and horizontal velocity components. (a) Downward pumping case. (b) Vector plot of the velocity profile along the side wall of the cylinder for the downward pumping case. (c) Upward pumping case. (d) Vector plot for the upward pumping case. For the downward pumping case, the horizontal velocity component is more pronounced than in the upward pumping case; the vertical velocity component is bigger for the upward pumping case. The vertical velocity component depends on the direction of rotation of the propeller, the horizontal component does not.

7. Y. Kolesnikov, C. Karcher, and A. Thess, Metall. Mater. Trans. B 42, (2011) 441

8. V. Minchenya, C. Karcher, Y. Kolesnikov, and A. Thess, Magnetohydrodynamics 45, (2009) 459

9. A. Wegfraß, C. Diethold, M. Werner, T. Fröhlich, B. Halbedel, F. Hilbrunner, C. Resagk, A. Thess, Appl. Phys. Lett. 100, (2012) 194103

10. C. Heinicke, S. Tympel, G. Pulugundla, I. Rahneberg, T. Boeck, and A. Thess, J. Appl. Phys. 112, (2012) 124914
11. C. Heinicke, Local Lorentz Force Velocimetry for liquid metal duct flows ( $\mathrm{PhD}$ thesis, Ilmenau University of Technology, 2013)

12. C. Heinicke, Exp. Fluids 54, (2013) 1560 